\title{
Analytic Formulas for Current Density Profiles in Small-Orbit Spiraling Electron Beams
}

\author{
J. P. Calame, Member, IEEE
}

\begin{abstract}
Analytic expressions for the current density as a function of radius in small-orbit, spiraling electron beams, including guiding center spread, are derived using probability theory. The expressions are valid for beams with a small spread in Larmor radius compared to the guiding center spread and a space charge depression below $10 \%$, which are typical for beams produced by modern magnetron injection guns. The current density profile is shown to exhibit a number of functional forms, depending on the relative size of the spread in guiding center radii compared to the average Larmor radius. Several applications for the new expressions are explored, including the calculation of the thermal loading experienced by beam-intercepting metal objects, as well as the computation of average electron density per unit volume (used in predicting the growth of electrostatic cyclotron instabilities). The analytic results are extended, in the vicinity of the beam edges, to include the effects of Larmor radius spread. The edge expressions are employed to predict the amount of current intercepted by a tunnel that closely surrounds the beam, which can be useful in selecting radial clearances.
\end{abstract}

Index Terms-Beam interception, electron beam profile, electrostatic cyclotron instability, magnetron injection gun.

\section{INTRODUCTION}

$\mathbf{S}$ MALL-ORBIT spiraling electron beams are employed as the energy source for the generation or amplification of electromagnetic radiation in gyro devices. Such devices include oscillators, which are used to produce nearly steady-state power levels exceeding $1 \mathrm{MW}$ in the millimeter-wave regime [1]-[6]. gyro oscillators are also used to heat materials during microwave processing [2], [7], [8]. Similarly, gyroklystron amplifiers are under intense development for use in millimeter-wave radar transmitters [2], [4], [9], and as a power source for large electron-positron colliders [2], [10].

The spiraling electron beams employed in these gyro devices are typically produced by an axis-symmetric magnetron injection gun (MIG) [11]-[13]. In its simplest form, an MIG consists of a slanted annular cathode immersed in an axial magnetic field, and surrounded by a funnel-shaped anode. During operation, the combined radial and axial electric field between the cathode and the anode obliquely accelerates the emitted electrons, but the magnetic field forces the electrons to miss the anode and begin spiraling as they move downstream. The electrons experience additional axial acceleration from the anode, and finally enter a region of increasing axial magnetic field to increase the perpendicular velocity (at the expense of axial ve-

Manuscript received May 24, 2000; revised October 10, 2000. This work was supported by the Office of Naval Research.

The author is with the Naval Research Laboratory, Washington, DC 20375

USA(e-mail: calame@mmace.nrl.navy.mil).

Publisher Item Identifier S 0093-3813(01)02894-6.

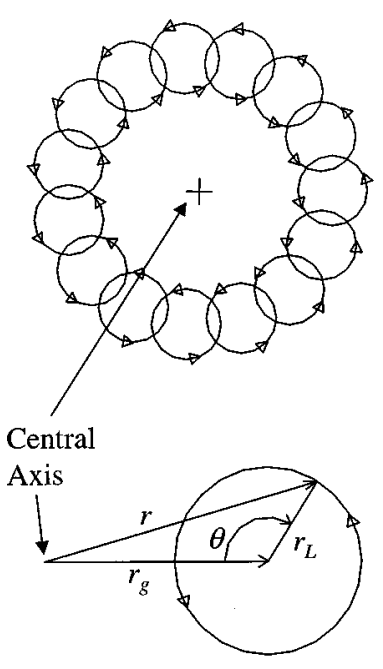

Fig. 1. Geometry of a small-orbit spiraling electron beam, and definitions of parameters that describe a single beamlet.

locity). At the output of the gun, and within the attached gyro device where there is a strong, uniform axial magnetic field, the electron beam has the geometry pictured in Fig. 1. The beam in Fig. 1 is classified as small-orbit because the radius of electron rotation, i.e., the Larmor radius $r_{L}$, is smaller than the guiding center radius $r_{g}$.

The properties of such beams, including the mean values and spreads of $r_{g}, r_{L}$, total energy, and velocity components, are simulated with particle trajectory codes [14]-[21]. The results of such simulations are used to refine the gun design, and are ultimately employed in the simulation and design of the gyro device. While the gyro device simulations usually proceed by test-particle methods, and include the spreads with a limited number of discordant electrons, there are several practical applications for which the functional form of the beam current density profile, $J(r)$, is needed. These applications include the interpretation of experimental radial profiles obtained from beam analyzers, and the prediction of temperature profiles along metallic objects that intercept the beam. Other applications include the computation of mean volumetric electron density (for estimating the growth of electrostatic cyclotron waves), and the calculation of the current intercepted by a tunnel that closely surrounds the beam.

The purpose of this paper is to develop improved analytic expressions for the radial current density profiles of small-orbit, spiraling electron beams, by means of analytic geometry and a purely ballistic model of the electron motion. In Section II, the inadequacy of commonly used, simple models of current density profile will be illustrated with some examples. Then, in 
Section III, the improved analytic formulas for the profile that take into account an arbitrary spread in guiding the center radius will be derived. A number of limitations and applications for the new formulas will be explored in Section IV, including the issue of a simultaneous spread in the Larmor radius.

\section{Simple Models of CURRENT Density}

Two common models used to describe the current density profile of a small-orbit beam are the uniform annulus model, and the perfect turning-point model [22]. The uniform annulus model assumes that the current density is constant within the annular region located between the orbit turning points of $r_{g}-r_{L}$ and $r_{g}+r_{L}$. This simple model has

$$
J(r)=\frac{I}{4 \pi r_{g} r_{L}}
$$

within the annulus and zero outside, where $I$ is the total beam current. In contrast, the perfect turning-point model is derived from the geometry of Fig. 1, in which

$$
r=\sqrt{r_{g}^{2}+r_{L}^{2}-2 r_{g} r_{L} \cos \theta}
$$

where $\theta$ is the gyrophase angle of the electron. The gyrophase angle is assumed to be a uniformly distributed random variable on the domain from 0 to $2 \pi$, so it has a probability distribution function, $\operatorname{pdf}(\theta)=(2 \pi)^{-1}$, within the domain. To find the radial current density profile under this assumption, one has to first compute the $\operatorname{pdf}(r)$ where $r$ is the electron radius computed with (2). One can write

$$
\operatorname{pdf}(r)=\frac{d}{d r}(\operatorname{cdf}(r))
$$

where $\operatorname{cdf}(r)$ is the cumulative distribution function [23] of the radius, which is the fraction of electrons with a radius less than or equal to $r$. The range of $\theta$ that satisfies this condition, denoted by $\Delta \theta$, can be found by solving (2) for $\theta$. Under the assumption that $\theta$ is uniformly distributed, the cdf is equal to $\Delta \theta /(2 \pi)$, or

$$
\operatorname{cdf}(r)=\frac{1}{\pi} \cos ^{-1}\left(\frac{r_{g}^{2}+r_{L}^{2}-r^{2}}{2 r_{L} r_{g}}\right)
$$

where $r_{g}-r_{L}<r<r_{g}+r_{L}$. The cdf is 0 for values of $r$ below this range, and 1 for values of $r$ that exceed the range. Upon differentiating, one obtains

$$
\operatorname{pdf}(r)=\frac{2 r}{\pi \sqrt{\left(\left(r_{g}+r_{L}\right)^{2}-r^{2}\right)\left(r^{2}-\left(r_{g}-r_{L}\right)^{2}\right)}}
$$

for $r_{g}-r_{L}<r<r_{g}+r_{L}$ and zero, otherwise. Note that this pdf is normalized so that $\int \operatorname{pdf}(r) d r=1$, where the differential element is just $d r$, and not the usual $r d r$ of cylindrical geometry (the extra factor of $r$ is actually part of the pdf, which avoids having to keep track of special radial normalization integrals in derivations with many different pdfs). Under this convention the current density profile is related to the $\operatorname{pdf}(r)$ by

$$
J(r)=\frac{I \mathrm{pdf}(r)}{2 \pi r} .
$$
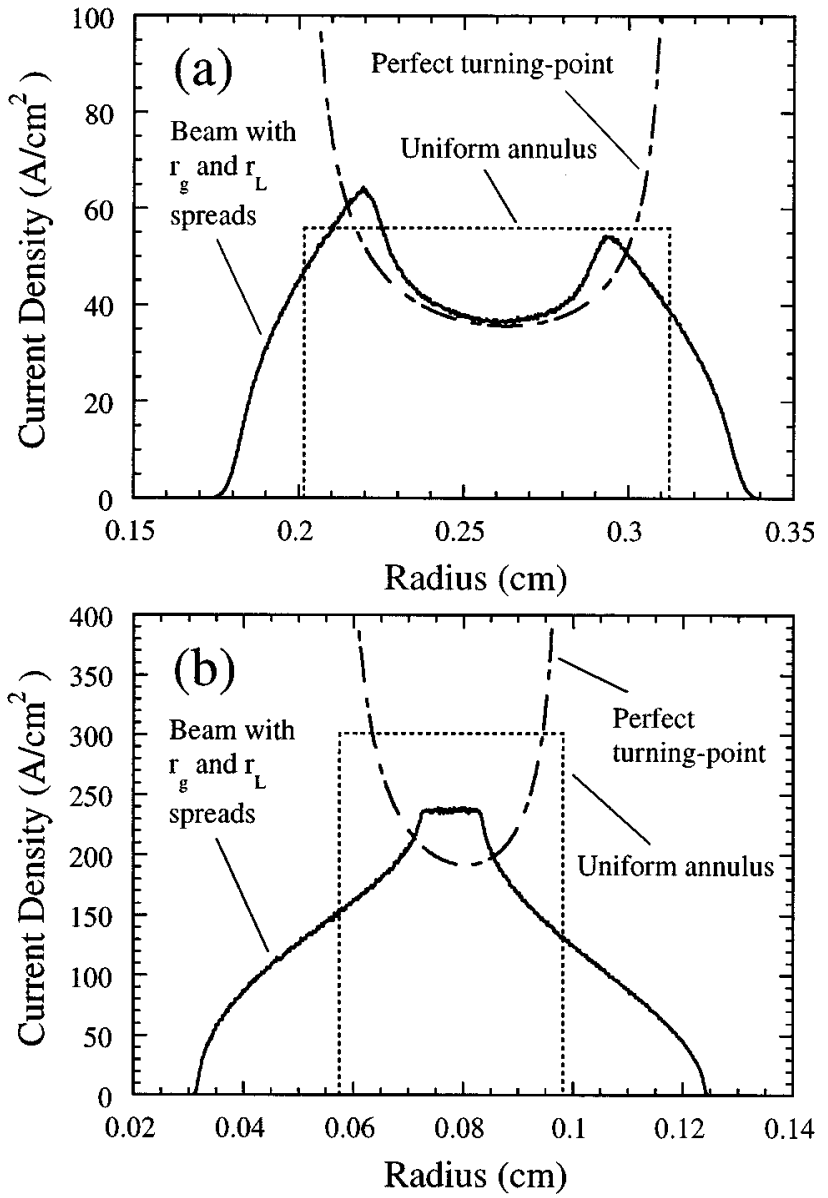

Fig. 2. Beam-optics code simulated current density profiles (solid lines) of (a) a beam used in a 35-GHz gyroklystron, and (b) a beam used in a high-average power, 94-GHz gyroklystron. The predictions of the uniform annulus model and the perfect turning point model are shown in each case.

One, therefore, obtains

$$
J(r)=\frac{I}{\pi^{2} \sqrt{\left(\left(r_{g}+r_{L}\right)^{2}-r^{2}\right)\left(r^{2}-\left(r_{g}-r_{L}\right)^{2}\right)}}
$$

for the perfect turning-point model when $r_{g}-r_{L}<r<r_{g}+r_{L}$, and zero, otherwise. This profile has asymptotes at the turning points, due to the stationary nature of the radial motion. It is similar to the classical probability distribution of a one-dimensional harmonic oscillator [24], but with some added complexity from the cylindrical geometry. A Cartesian version of the perfect turning-point model, with $r_{g} \rightarrow \infty$, was investigated by Bondeson and Antonsen during calculations of electrostatic cyclotron instability growth [22].

Comparisons between these models and the current density profiles of beams created by two different magnetron injection guns are shown in Fig. 2. The first beam, Fig. 2(a), has a current of $10 \mathrm{~A}$, and was used in a number of $35-\mathrm{GHz}$ gyroklystron experiments [25], [26], while the beam of Fig. 2(b) has a current of $6 \mathrm{~A}$, and was employed in a 94-GHz (W-band), high average-power gyroklystron [27]. The plotted current density profiles are based on Electron GUN (EGUN) [14], [15] simulations of these guns [25], [28], which include space charge effects. Either 64 or 128 rays were used to compute the median guiding 
center radius $r_{g 0}$, the mean Larmor radius $r_{L 0}$, and the respective distributions of electrons about $r_{g 0}$ and $r_{L 0}$ (the guiding center and Larmor spreads). The use of the median value of $r_{g}$, rather than the mean, will be explained in the next section of the paper. Next, the $\operatorname{pdf}(r)$ was obtained by Monte Carlo evaluation of (2) and a histogram-based counting scheme, assuming a uniform distribution of $\theta$, and the EGUN-derived distributions of $r_{g}$ and $r_{L}$. Finally, the current density profile was computed with (6). One can see from the plots that the simple models of $J(r)$ from (1) and (7), in which $r_{g}$ and $r_{L}$ have been set equal to $r_{g 0}$ and $r_{L 0}$, bear little resemblance to the simulated profiles as a result of the spreads present on the beams. The need for improvements to these simple models, while maintaining the analytic formulation as much as possible, is evident.

\section{IMPROVED ANALYTIC EXPRESSIONS FOR CURRENT DENSITY}

The first step in developing better descriptions of the current density profiles is to include the effects of a spread in the guiding center radii. Although realistic beams have spread in both the guiding centers and Larmor radii, the spread in Larmor radii from modern magnetron injection guns is generally small, with $\sigma_{L} / r_{L 0}$ below $10 \%$. In this notation, $\sigma_{L}$ is the standard deviation of the Larmor radius. The beams of Fig. 2(a) and (b) have $\sigma_{L} / r_{L 0}$ of $5 \%$ and $1.5 \%$, respectively. The low spread is mandated by the requirements of gyro amplifiers, which suffer severely in performance for larger values of Larmor spread [29], [30]. Even in gyro oscillators, which have a less severe requirement, the guns are almost always designed with Larmor spreads of $10 \%$ or less [13]. Conversely, modern magnetron injection guns typically have a significant guiding center spread, often comparable to, or exceeding, the mean Larmor radius. Guiding center spread is further increased by the use of a steep cathode angle relative to the magnetic field lines in the gun. This is done to create a laminar beam, which reduces the effect of space charge in the beam compression region between the cathode and the microwave circuit, allowing the creation of a low velocity-spread beam [28].

The distribution of the guiding center radii in a typical MIG beam, described by a probability distribution $\operatorname{pdf}\left(r_{g}\right)$, is not uniformly distributed, but instead is very closely described by a radially weighted trapezoidal distribution

$$
\operatorname{pdf}\left(r_{g}\right)= \begin{cases}\frac{r_{g}}{2 a_{g} r_{g 0}}, & \left|r_{g}-r_{g 0}\right| \leq a_{g} \\ 0, & \text { else. }\end{cases}
$$

In this distribution $r_{g 0}$ is the median guiding center radius (midpoint of the trapezoid), as opposed to the mean, and $a_{g}$ defines the extent of the distribution on either side of the median. EGUN simulations of the distribution of guiding center radii for the two MIG beams discussed in Section II are shown in Fig. 3, along with a forced linear fit through the origin (proportional to $r_{g}$ ) for each case. The good agreement between the simulations and the functional form of (8) is apparent.
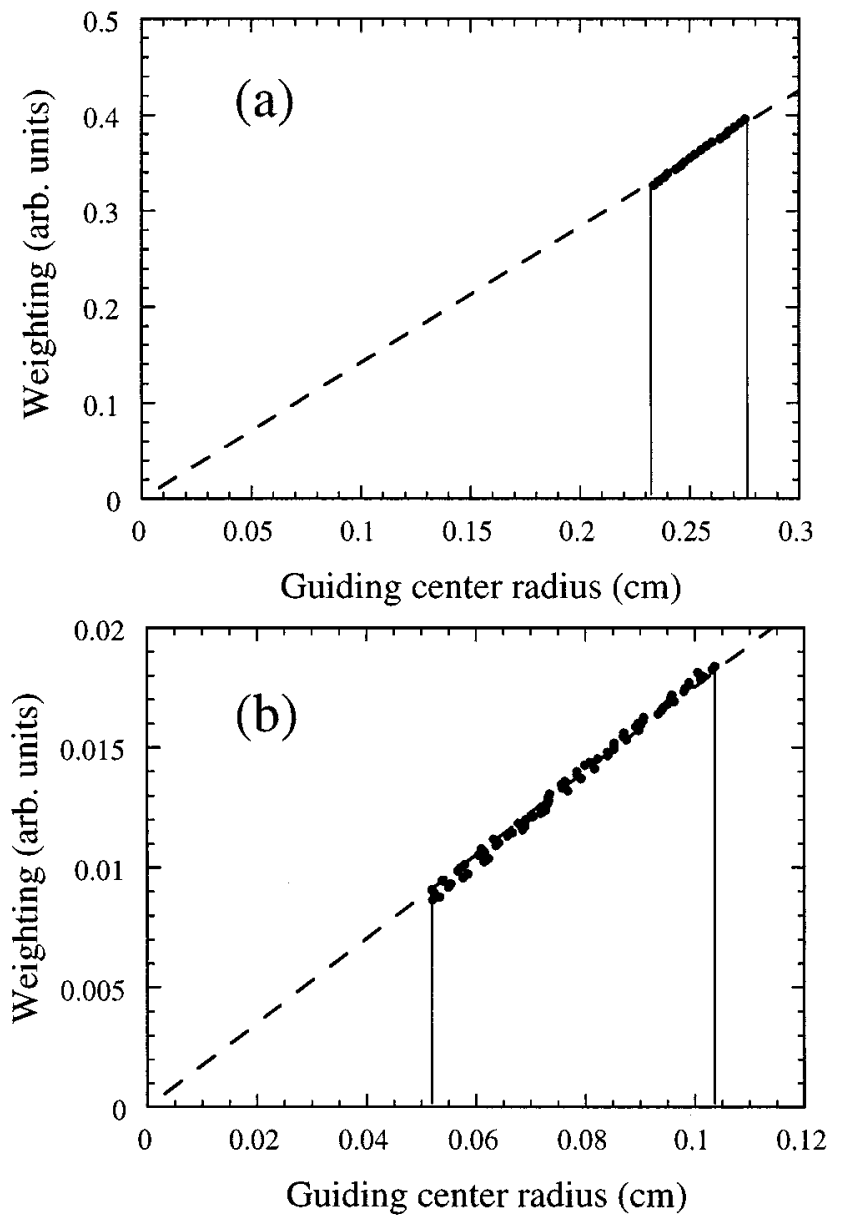

Fig. 3. Beam-optics code simulated distribution of guiding center radii (dots) for the (a) 35-GHz gyroklystron gun and (b) 94-GHz gyroklystron gun. Also shown in each case are radially-weighted fits through the data (dashed lines).

The radially weighted distribution arises as a result of geometry and canonical angular momentum conservation. From canonical angular momentum conservation, each electron has

$$
\left(r_{g}^{2}-r_{L}^{2}\right) B_{z}=r_{c}^{2} B_{z c}
$$

where

$B_{z} \quad$ uniform axial magnetic field at the output end of the gun;

$r_{c}$ radius on the cathode from which the electron was emitted;

$B_{z c} \quad$ axial magnetic field at the point of emission.

In most small-orbit beams, $\left(r_{L} / r_{g}\right)^{2} \ll 1$, and the axial magnetic field along the cathode surface is fairly constant, so the guiding center radius of an electron is (nearly) directly proportional to the cathode radius at the emission site [28]. For a uniform emission current density on the cathode, the number of electrons that are emitted from an incremental range of cathode radii $r_{c}$ to $r_{c}+d r_{c}$ is proportional to $r_{c}$, from simple geometric considerations. Therefore, the pdf of guiding center radius is proportional to $r_{g}$, within the limits of the approximations.

To derive the current density profiles for a beam with this type of guiding center spread and zero Larmor spread $\left(r_{L}=\right.$ $r_{L 0}$ ), one must consider the behavior of the electron radius, (2), for a uniform distribution of gyrophase angle, and the radially 


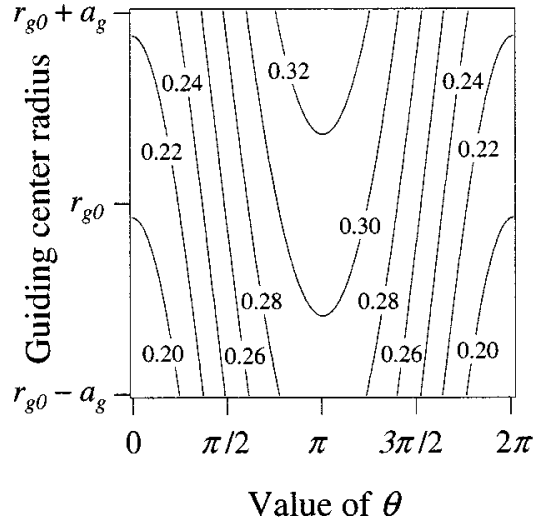

Fig. 4. A plot of level curves of an electrons radial coordinate $(r)$, as a function of gyrophase angle $(\theta)$, and guiding center radius. For this example, $r_{g 0}=$ $0.257 \mathrm{~cm}, a_{g}=0.0212 \mathrm{~cm}$, and $r_{L}=0.0554 \mathrm{~cm}$.

weighted distribution of guiding center radius. The derivation is somewhat similar to the perfect turning-point case in Section II, except now the electron radius is a function of the two random variables [23] instead of just one. A plot of the level curves of radius, as given by (2) as a function of $\theta$ and $r_{g}$, is shown in Fig. 4, where $r_{g 0}$ has been fixed at $0.257 \mathrm{~cm}$ and $r_{L}$ at 0.0554 $\mathrm{cm}$. These two values are associated with the $35-\mathrm{GHz}$ electron gun discussed above and in Fig. 2(a), which will be used as an example throughout the rest of the paper. For the plot in Fig. 4, the value of $a_{g}$ has been set at an EGUN-derived value of 0.0212 $\mathrm{cm}$. It is important to note that $a_{g}$ is smaller than $r_{L}$ in making this plot. The $\operatorname{cdf}(r)$ can be evaluated as the fraction of electrons with radius $\leq r$, which is given by an integral of the form

$$
\operatorname{cdf}(r)=\int_{0}^{2 \pi} d \theta \operatorname{pdf}(\theta) \int_{r_{g 0}-a_{g}}^{r_{g 0}+a_{g}} d r_{g} \operatorname{pdf}\left(r_{g}\right) f\left(r, \theta, r_{g}\right)
$$

where $f=1$ if the electron described by $\theta$ and $r_{g}$ has a radius $\leq r$, and $f=0$ if the radius exceeds $r$. Thus, the cdf is essentially a weighted area in $\theta, r_{g}$ space. The separatrix between the two states of $f$ in $\theta, r_{g}$ space is given by the level curve of the radius, as in Fig. 4, given by

$$
r_{g}=r_{L} \cos \theta+\sqrt{r^{2}-r_{L}^{2} \sin ^{2} \theta}
$$

To write the integral out explicitly, one first notes that the level curves of Fig. 4 are symmetric around $\theta=\pi$, so the examination of the separatrix and, thus, the integration in $\theta$, can be restricted from 0 to $\pi$, with integrated result subsequently multiplied by two.

For values of $\mathrm{r}$ below $r_{g 0}-a_{g}-r_{L}$, the cdf is zero by simple consideration of the geometry. For values of $r$ somewhat greater than this lower limit, the separatrix (level curve) begins on the $\theta=0$ axis at $r_{g}=r+r_{L}$, and pursues a concave downward path to intercept the $r_{g}=r_{g 0}-a_{g}$ boundary at an angle

$$
\theta_{2}=\cos ^{-1}\left[\frac{\left(r_{g 0}-a_{g}\right)^{2}+r_{L}^{2}-r^{2}}{2\left(r_{g 0}-a_{g}\right) r_{L}}\right]
$$

found by solving (11). Under these conditions, one can write out (10) as

$$
\begin{aligned}
& \operatorname{cdf}(r)= \\
& \begin{array}{r}
\frac{1}{4 \pi a_{g} r_{g 0}} \int_{0}^{\theta_{2}}\left(r_{L}^{2} \cos ^{2} \theta+2 r_{L} \cos \theta \sqrt{r^{2}-r_{L}^{2} \sin ^{2} \theta}\right. \\
\left.+r^{2}-r_{L}^{2} \sin ^{2} \theta-\left(r_{g 0}-a_{g}\right)^{2}\right) d \theta
\end{array}
\end{aligned}
$$

where the $\operatorname{pdf}(\theta)$ has been assumed to be uniformly distributed over $2 \pi, \operatorname{pdf}\left(r_{g}\right)$ is given by the radially weighted distribution of (8), and the integration in $r_{g}$ was carried out from $r_{g 0}-a_{g}$ to the value given by (11). As in the perfect turning-point case of Section II, the $\operatorname{pdf}(r)$ is found by differentiating the cdf with respect to $r$. Because the lower limit of the integration is fixed at zero, and the value of the integrand at $\theta=\theta_{2}$ is also zero, one can simply bring the differentiation inside the integral and write

$$
\begin{aligned}
& \operatorname{pdf}(\mathrm{r})=\frac{1}{2 \pi \mathrm{ag}_{\mathrm{g}} \mathrm{r}_{\mathrm{g} 0}} \\
& \quad \times \int_{0}^{\theta_{2}} r\left(1+\frac{r_{L} \cos \theta}{\sqrt{r^{2}-r_{L}^{2} \sin ^{2} \theta}}\right) d \theta .
\end{aligned}
$$

The integral can be evaluated analytically to give

$$
\operatorname{pdf}(r)=\frac{r}{2 \pi a_{g} r_{g 0}}\left[\theta_{2}+\sin ^{-1}\left(\frac{r_{L}}{r} \sin \theta_{2}\right)\right] .
$$

This equation remains valid as $r$ increases, in what shall be called Region 1, until the left-hand $(\theta=0)$ intercept of the separatrix reaches the maximum permissible value, at $r_{g}=r_{g 0}+a_{g}$. This occurs when $r=r_{g 0}+a_{g}-r_{L}$. For larger values of radius, the separatrix has the upper boundary value of $r_{g}=r_{g 0}+a_{g}$ at $\theta$ given by

$$
\theta_{1}=\cos ^{-1}\left[\frac{\left(r_{g 0}+a_{g}\right)^{2}+r_{L}^{2}-r^{2}}{2\left(r_{g 0}+a_{g}\right) r_{L}}\right]
$$

and dives downward to reach the $r_{g}=r_{g 0}-a_{g}$ boundary at $\theta_{2}$. For this case, range of integration in computing the cdf as in (13) is now $\theta_{1}$ to $\theta_{2}$, with an extra term added to the cdf resulting from the radially weighted area in $\theta, r_{g}$ space located between 0 to $\theta_{1}$ and $r_{g 0} \pm a_{g}$. However, when differentiating the cdf to obtain the pdf, one must remember in general that

$$
\begin{aligned}
\frac{d}{d r} \int_{\theta_{1}}^{\theta_{2}} g(\theta, r) d \theta & =\int_{\theta_{1}}^{\theta_{2}} \frac{\partial}{\partial r} g(\theta, r) d \theta \\
& +g\left(\theta_{2}, r\right) \frac{d \theta_{2}}{d r}-g\left(\theta_{1}, r\right) \frac{d \theta_{1}}{d r}
\end{aligned}
$$

where $g$ is the integrand of (13), and in this instance the $d \theta_{1} / d r$ term exactly cancels the effect of the extra area [note that the two extra terms in (17) were both zero in the previous calculation]. Working similarly as before, one ultimately obtains

$$
\begin{array}{r}
\operatorname{pdf}(r)=\frac{r}{2 \pi a_{g} r_{g 0}}\left[\theta_{2}-\theta_{1}+\sin ^{-1}\left(\frac{r_{L}}{r} \sin \theta_{2}\right)\right. \\
\left.-\sin ^{-1}\left(\frac{r_{L}}{r} \sin \theta_{1}\right)\right] .
\end{array}
$$


TABLE I

RADIAL Locations of THE REgIONS IN THE CURRENT DENSITY PROFILE

\begin{tabular}{l|c|c|c}
\hline \multicolumn{1}{c|}{ Case } & Region 1 & Region 2 & Region 3 \\
\hline I. $a_{g}<r_{L}$ & $r_{g 0}-a_{g}-r_{L} \leq r<r_{g 0}+a_{g}-r_{L}$ & $r_{g 0}+a_{g}-r_{L} \leq r<r_{g 0}-a_{g}+r_{L}$ & $r_{g 0}-a_{g}+r_{L} \leq r \leq r_{g 0}+a_{g}+r_{L}$ \\
\hline II. $a_{g}=r_{L}$ & $r_{g 0}-2 r_{L} \leq r<r_{g 0}$ & Does not exist & $r_{g 0} \leq r \leq r_{g 0}+2 r_{L}$ \\
\hline III. $a_{g}>r_{L}$ & $r_{g 0}-a_{g}-r_{L} \leq r<r_{g 0}-a_{g}+r_{L}$ & $r_{g 0}-a_{g}+r_{L} \leq r<r_{g 0}+a_{g}-r_{L}$ & $r_{g 0}+a_{g}-r_{L} \leq r \leq r_{g 0}+a_{g}+r_{L}$ \\
\hline
\end{tabular}

Case I
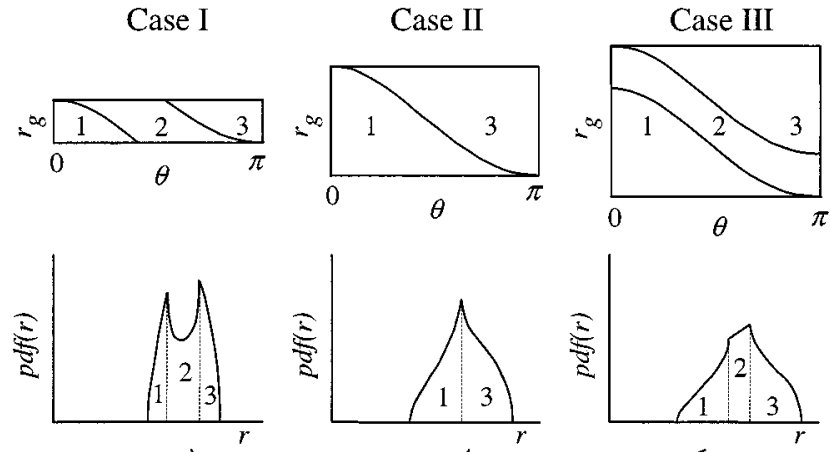

Fig. 5. Diagrams of the behavior of the separatrix (top row) in $\theta, r_{g}$ space and the corresponding shapes of the probability distribution function of radius, $\operatorname{pdf}(r)$ (middle row), for Cases I-III conditions. The locations of Regions 1-3 within the diagrams are shown with the Arabic numerals. The radial locations of these regions versus guiding center spread $a_{g}$ are shown in the bottom diagram.

This region (number 2) continues to be valid until the value of $\theta_{2}$ reaches $\pi$, which occurs when $r=r_{g 0}-a_{g}+r_{L}$. Beyond this transition the separatrix begins at $\theta=\theta_{1}$, when $r_{g}=r_{g 0}+a_{g}$, and drops with a concave upward curvature to intercept the $\theta=$ $\pi$ line at $r_{g}=r-r_{L}$. Pursuing a similar calculation to those above yields

$$
\operatorname{pdf}(r)=\frac{r}{2 \pi a_{g} r_{g 0}}\left[\pi-\theta_{1}-\sin ^{-1}\left(\frac{r_{L}}{r} \sin \theta_{1}\right)\right] .
$$

This region (number 3) remains valid until $r=r_{g 0}+a_{g}+$ $r_{L}$, at which point the pdf is zero for larger radii, by simple consideration of the geometry.

The preceding analysis is valid for relatively small guiding center spreads, when $a_{g}<r_{L}$, which will be referred to as Case I. The boundaries of the three regions for this case are listed in Table I, and a pictorial representation of the behavior of the separatrix, and the shape of the pdf, is shown on the left side of Fig. 5. As the amount of guiding center spread increases, the size of Region 2 decreases, and finally disappears when $a_{g}=r_{L}$. This is catalogued as Case II in Table I. Examining the center part of

Fig. 5, one can see that the left-hand $\theta=0$ intercept of the separatrix reaches the maximum permissible $r_{g}$ value of $r_{g 0}+a_{g}$ at the same time that the right-hand $\theta=\pi$ intercept reaches $r_{g}=r_{g 0}-a_{g}$. Finally, for even larger values of guiding center spread (Case III), when $a_{g}>r_{L}$, the separatrix reaches $\theta=\pi$ before the left hand intercept of the $\theta=0$ axis has reached $r_{g 0}+a_{g}$, a condition which is visible in the right part of Fig. 5 . Therefore, there is a new Region 2 that exists from this condition until the $\theta=0$ intercept of the separatrix finally reaches the $r_{g 0}+a_{g}$ upper bound, at which time the separatrix reverts to normal Region 3 behavior. The boundaries of the regions for Case III are listed in Table I. For the abnormal Region 2 in Case III, the pdf can be shown to be

$$
\operatorname{pdf}(r)=\frac{r}{2 a_{g} r_{g 0}}
$$

Combining the preceding expressions for the $\operatorname{pdf}(r)$ with $(6)$, one obtains the current density profiles given by

$$
J(r)=\frac{I}{4 \pi^{2} a_{g} r_{g 0}}\left(\theta_{2}+\sin ^{-1}\left(\frac{r_{L}}{r} \sin \theta_{2}\right)\right)
$$

in Region 1 for all cases,

$$
J(r)= \begin{cases}\frac{I}{4 \pi^{2} a_{g} r_{g 0}}\left(\theta_{2}-\theta_{1}+\sin ^{-1}\right. & \\ \left.\left(\frac{r_{L}}{r} \sin \theta_{2}\right)-\sin ^{-1}\left(\frac{r_{L}}{r} \sin \theta_{1}\right)\right), & \text { Case I } \\ \frac{I}{4 \pi a_{g} r_{g 0}}, & \text { Case III }\end{cases}
$$

in Region 2, and

$$
J(r)=\frac{I}{4 \pi^{2} a_{g} r_{g 0}}\left(\pi-\theta_{1}-\sin ^{-1}\left(\frac{r_{L}}{r} \sin \theta_{1}\right)\right)
$$

in Region 3 for all cases. The angles, $\theta_{1}$ and $\theta_{2}$, are functions of $r$ given previously in (16) and (12). The boundaries of the regions are shown graphically in the bottom portion of Fig. 5, in addition to the previous listing in Table I. The simple geometrical relations between the locations of the boundaries and the values of $r_{g 0}, a_{g}$, and $r_{L}$ are easily obtained from this diagram.

The radial current density profile takes on a variety of shapes depending on the value of $a_{g}$ in relation to $r_{L}$. Specific examples of current density profiles given by the analytic formulas are plotted in Fig. 6 for a number of guiding center spreads, where once again $r_{g 0}$ has been fixed at $0.257 \mathrm{~cm}$ and $r_{L}$ at 0.0554 $\mathrm{cm}$. The beam current is $10 \mathrm{~A}$ in each case. For small values of 


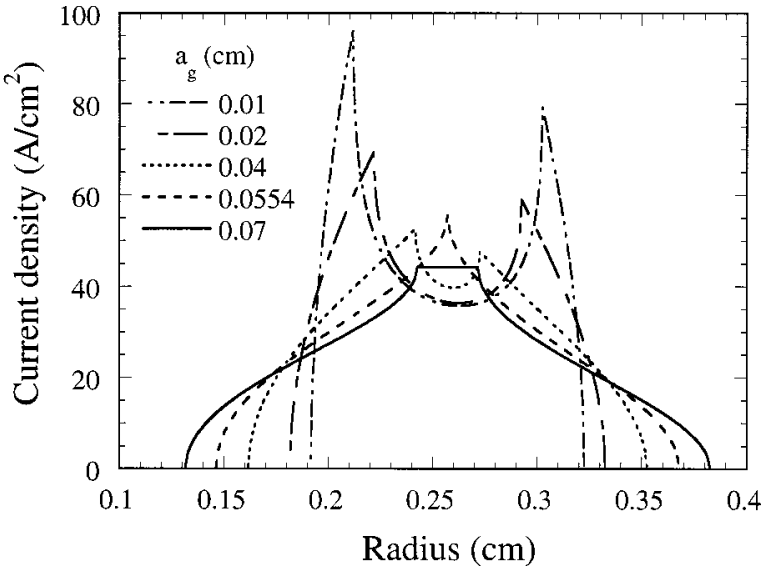

Fig. 6. Plots of current density versus radius for various values of guiding center spread $\left(a_{g}\right)$. These analytic calculations were performed with $r_{g 0}=$ $0.257 \mathrm{~cm}, r_{L}=0.0554 \mathrm{~cm}$, and 10-A beam current.

guiding center spread (Case I), one finds Region 2 dominating the overall profile, with the peaks in the current density profile located very close to the zero spread positions of $r_{g 0}-r_{L}$ and $r_{g 0}+r_{L}$. Within the center of Region 2, the functional form is, in fact, very similar to the perfect turning-point expression given by (7), except that the asymptotes near the turning points are rendered finite by the spread. As the value of $a_{g}$ increases, the peaks in the current density profile move inward toward the median guiding center radius, as well as decrease in magnitude. At the same time, the tails on the inside and outside edges of the beam, corresponding to Regions 1 and 3, respectively, increase in spatial extent. When the value of $a_{g}$ is equal to the Larmor radius, the central region does not exist, and the current density profile forms a cusp at $r=r_{g 0}$, with the Region 1 and 3 tails on the sides. Finally, for even larger values of guiding center spread, a central region of uniform current density occurs, surrounded by fully-developed tails. The functional forms of the current density profiles are visibly similar to the distributions in Fig. 2, in spite of the absence of Larmor radius spread in these analytic results. Direct comparisons between the EGUN-simulated beam profiles from Fig. 2 and the predictions of the new analytic formulas are shown in Fig. 7. One can see that Fig. 7(a) is a Case I profile ( $a_{g}$ of $0.0212 \mathrm{~cm}$ compared to $r_{L}$ of 0.0554 $\mathrm{cm}$ ), while Fig. 7(b) is described by Case III ( $a_{g}$ of $0.0259 \mathrm{~cm}$ compared to $r_{L}$ of $0.0204 \mathrm{~cm}$ ). Overall, the agreement in these two examples is excellent.

\section{DISCUSSION}

The analytic expressions for current density, (21)-(23), are subject to certain limitations that arise from the assumptions used in deriving them. The formulas do not include a spread in Larmor radius, which is always present to some extent due to a variety of phenomena [21], including: beam-optics aberrations; space charge distortions [28], [31]; cathode surface roughness [32], [33]; and the finite physical temperature [32] and temperature nonuniformities of the emitting surface. The formulas also ignore other, more subtle effects of space charge, since they are based on purely ballistic motions of the electrons
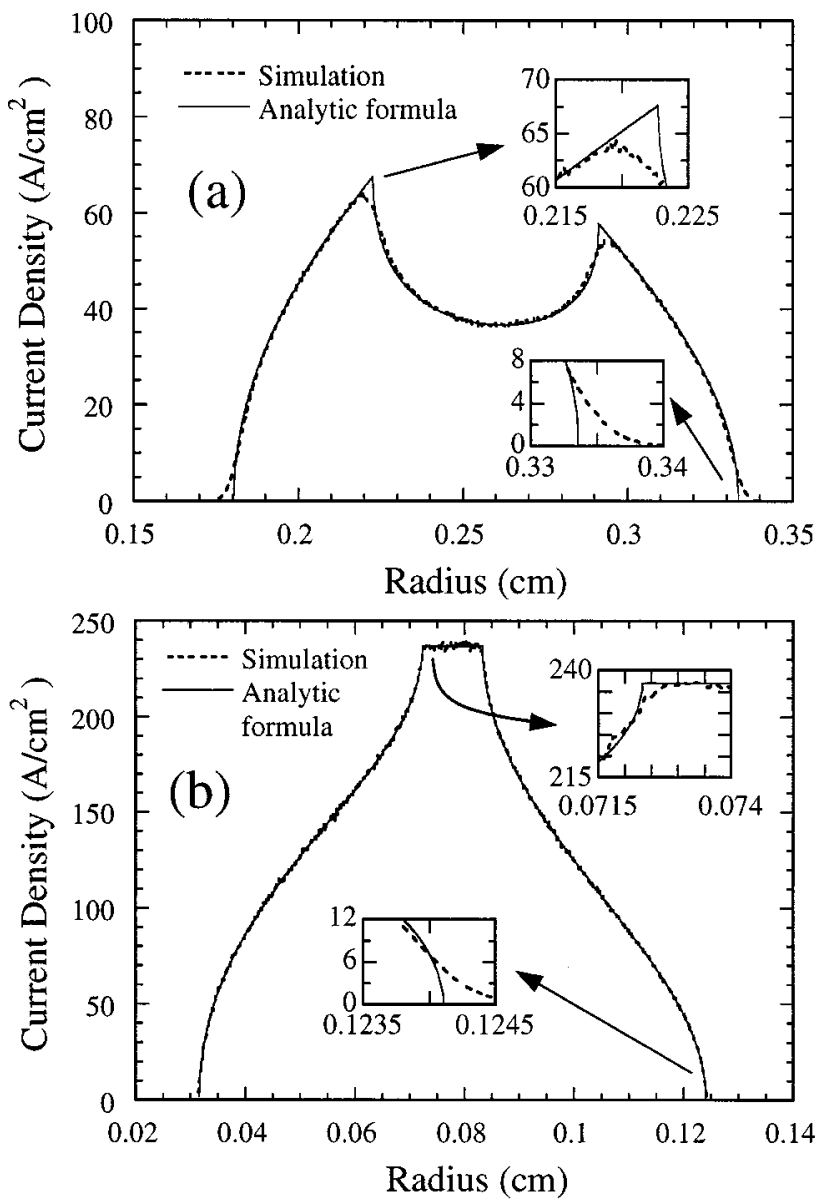

Fig. 7. Comparison between beam-optics simulated current density profiles from Fig. 2 (dashed lines) for (a) the beam used in a 35-GHz gyroklystron, and (b) the beam used in a high-average power, 94-GHz gyroklystron, and the profiles computed with the improved analytic formulas described in this paper (solid lines).

combined with the geometry of the orbits. Space charge potential depression will cause the time-averaged kinetic energy of an electron to depend on its guiding center radius, and this kinetic energy spread contributes to a Larmor radius spread that is not included. Furthermore, the space charge depression will affect an individual electron during its orbit around the guiding center. As the electron moves toward the center of the beam, the space charge potential will reduce the kinetic energy, slowing the electron's transverse velocity. This causes the pdf of the gyrophase angle $\theta$ to no longer be strictly uniform, and furthermore the distribution of $\theta$ is weakly dependent on $r_{g}$. Fortunately, in most beams used in gyrodevices, the effects of space charge are deliberately kept small, by either selecting a suitably high operating voltage, or by increasing the average guiding center radius, because space charge-induced velocity spread and related effects degrade the efficiency of gyroamplifiers and gyro oscillators. Generally, the space charge depression is kept below 5\% of the beam voltage for gyroamplifiers, and below $10 \%$ for gyro oscillators [13], [29], [30], [34]. Depressions of this magnitude have only a modest impact on the overall Larmor spread.

While some Larmor spread on the beam is unavoidable, it has a minimal impact on the shape of the current density profile if there is a coexisting, significant spread in guiding centers. 


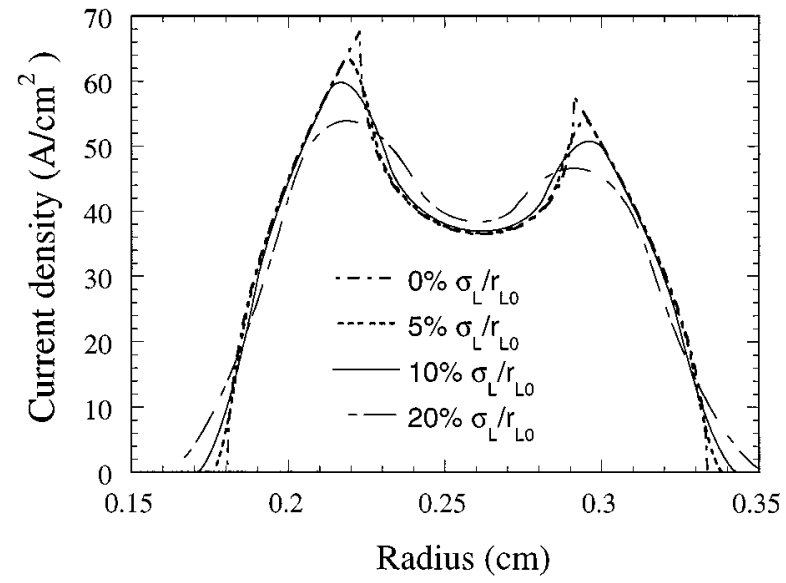

Fig. 8. Effect of a Gaussian Larmor radius spread on the current density profile, assuming $r_{g 0}=0.257 \mathrm{~cm}, a_{g}=0.0212 \mathrm{~cm}, r_{L 0}=0.0554 \mathrm{~cm}$, and 10 A beam current.

Some examples demonstrating the minimal impact are shown in Fig. 8. For these calculations, the median guiding center and Larmor radius were once again held at 0.257 and $0.0554 \mathrm{~cm}$, respectively, and $a_{g}$ was set at $0.0212 \mathrm{~cm}$, and the beam current was $10 \mathrm{~A}$. The distribution in Larmor radius was assumed to be a Gaussian with a standard deviation of $\sigma_{L}$. Unfortunately, the current density profile including Larmor spread cannot be written analytically, so (21)-(23) were numerically integrated over the $r_{L}$ distribution. Inspection of the figure shows modest reductions in the peak current densities, and the creation of small tails on the inside and outside edges of the beam. A quantitative discussion of the tails at the beam edges as a function of the Larmor spread will be presented at the end of this section. Nevertheless, the agreement with the zero-Larmor spread formula is excellent (in this example) for $\sigma_{L} / r_{L 0}=5 \%$ and adequate for $\sigma_{L} / r_{L 0}=10 \%$. For a large spread, $\sigma_{L} / r_{L 0}=20 \%$, the agreement is poor.

However, to establish a more general understanding of the effect of Larmor spread on the most sensitive part of the profile, which is the region of peak current density (near $r=r_{g 0}+$ $a_{g}-r_{L 0}$ ), a systematic study was performed. A set of Case I profiles was examined in a numerical grid search over a wide range of $r_{g 0}, a_{g}, r_{L 0}$, and $\sigma_{L}$, and compared to the analytic expressions. The zero-Larmor spread profile formulas [(21)-(23)] were found to overestimate the actual peak current densities by a percentage amount given by

$$
\text { error } \approx\left(\frac{r_{L 0}}{a_{g}}\right)\left(\frac{\sigma_{L}}{r_{L 0}}\right) \times 50 \%
$$

where $r_{L 0}$ has been deliberately left uncanceled in the equation to retain the fractional Larmor spread notation, $\sigma_{L} / r_{L 0}$. This presentation also emphasizes that the error is inversely proportional to the guiding center spread relative to the mean Larmor radius, $a_{g} / r_{L 0}$ (as opposed to the fractional spread in guiding centers, $\left.a_{g} / r_{g 0}\right)$. For the beams pictured in Fig. 8, (24) predicts peak current errors of $6.5 \%, 13 \%$, and $26 \%$ for $\sigma_{L} / r_{L 0}$ equal to $5 \%, 10 \%$, and $20 \%$, respectively, which are in good agreement with the results in the figure. More generally, the error formula allows a rapid, worst-case assessment of the applicability of the current density profile formulas for Case I profiles, which are the most sensitive to Larmor spread. For Case III profiles, where $a_{g}$ exceeds $r_{L 0}$ [and (24) is no longer applicable], the addition of Larmor spread does not generally affect the peak current density. Instead, the Larmor spread softens the boundaries of the uniform current region, as is visible in Fig. 7(b), in addition to extending the outer tails of the beam.

Even though they lack the subtleties of Larmor spread, the analytic expressions for current density, (21)-(23), are directly useful for a number of purposes. For example, if a radial current density profile is experimentally measured with a scanning beam analyzer, the analytic formulas could be used in a curve fitting procedure to arrive at experimental values of mean Larmor, guiding center radii, and the guiding center spread. Accurate measurements of these parameters are useful for confirming the predictions of beam optics codes. A second important application of the current density profiles occurs in the thermal modeling of beam-intercepting support rods used in coaxial gyro amplifiers [35], [36] or ubitrons [37]. In high average power applications, the inner member of the rf interaction structure is typically suspended from beyond the beam dump, often in conjunction with an inverted magnetron injection gun configuration, to avoid beam interception on the support structure [38]. The inner member can also be supported from the cathode by an insulator to avoid beam interception [39]. However, in high peak power, low average power proof-of-principle devices, radial pins placed directly in the path of the beam are typically used for support.

The average heating of such support pins can be kept as small as required, simply by reducing the repetition rate. However, the heating that occurs within a single beam pulse cannot be reduced by this technique, so a knowledge of the peak current density in the beam is critical to determining if localized melting is a danger for a given pin material. The radial temperature profile $T(r)$ induced by a pulsed electron beam impacting a material is given by

$$
T(r)=T_{0}+\left(\frac{V \tau}{\rho_{m} c_{p} R_{d}}\right) J(r)
$$

where

$T_{0} \quad$ starting temperature;

$V$ beam voltage;

$\tau \quad$ pulselength;

$\rho_{m} \quad$ mass density;

$c_{p} \quad$ specific heat;

$R_{d} \quad$ (voltage-dependent) electron penetration depth into the material.

The penetration depth versus voltage can be looked up in tables [40]. Refractory metals such as tungsten or molybdenum are the materials of choice for this application by virtue of their very high melting points, in spite of the small electron penetration depths into these dense materials. With the aid of (25), the single-pulse temperature profile along the support pin, or along any beam intercepting object, can be calculated using the analytic current density profile formulas. Referring to Fig. 6, the most concentrated current densities occur in the Case I and II profiles, at the points $r_{g 0}+a_{g}-r_{L}$ and $r_{g 0}-a_{g}+r_{L}$, while Case III has the maximum throughout its Region 2. Although (25) neglects thermal diffusion effects that occur during 


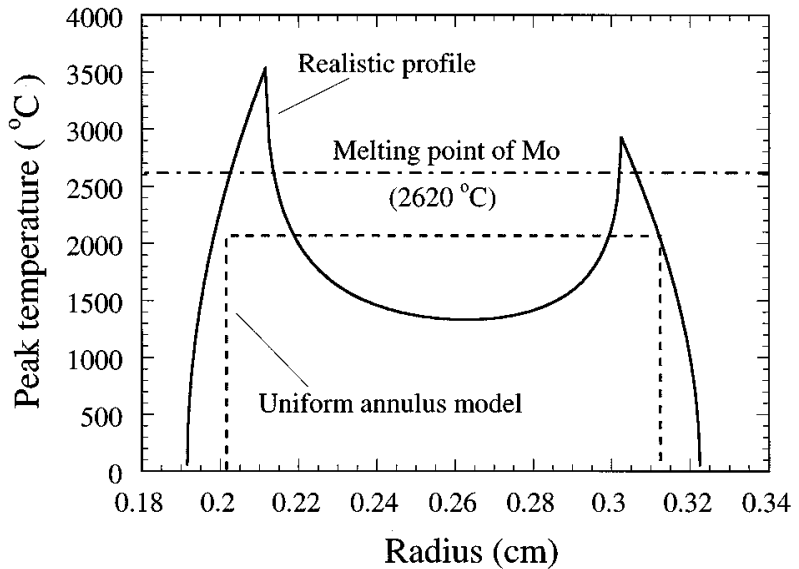

Fig. 9. An example thermal profile calculations along a beam-intercepting, molybdenum pin for a $10-\mathrm{A} 70-\mathrm{kV}$ beam with $r_{g 0}=0.257 \mathrm{~cm}, a_{g}=0.01$ $\mathrm{cm}, r_{L 0}=0.0554 \mathrm{~cm}$, and a $2 \mu \mathrm{s}$ pulselength. The realistic profile (solid line) predicts that melting will occur at this pulselength, while the uniform annulus model does not.

the pulse, and ignores the long term disruptive effects of extreme thermal shocks, it is useful as a starting point in choosing a pin material or setting a maximum pulselength. The result of such a calculation is shown for a molybdenum pin in Fig. 9, using the analytic profile (21)-(23) for a beam with $r_{g 0}, r_{L 0}$, and $a_{g}$ equal to $0.257,0.05544$, and $0.01 \mathrm{~cm}$, respectively, along with (25). The beam voltage for the computation was $70 \mathrm{kV}$, and the pulselength was $2 \mu \mathrm{s}$. The molybdenum is predicted to melt under these circumstances, while the same computation performed with the simple uniform annulus model does not predict melting. The refined formulation indicates that the pulselength must be reduced below $1.5 \mu$ s to avoid melting. An example of a more advanced calculation that includes thermal conductivity effects is described in [35].

A third important application of the current density profiles concerns estimating the average electron density per unit volume within the beam, which is important for computing the spatial growth rate of electrostatic cyclotron instabilities [22]. Such instabilities have been predicted to degrade beam quality [41], [42], thereby reducing device efficiency, or lead to the growth of shot noise which can hamper gyro amplifier performance in radar and communications systems [43], [44]. The growth rate of electrostatic instabilities scales as the electron plasma frequency, which is proportional to the square root of the mean beam density. For a beam with small axial velocity spread, the local density profile is related to the current density by

$$
n(r)=\frac{J(r)}{e \mathrm{v}_{z}}
$$

where $e$ is the electron charge and $\mathrm{v}_{z}$ is the axial beam velocity. In studying electrostatic cyclotron instabilities [22], the simple uniform annulus model (1) is one common choice for calculating an average current density. This is completely appropriate for beams with small guiding center spread and $r_{L} / r_{g 0} \ll 1$. However, this approximation is not reasonable for beams with significant guiding center spread, as shown in Sections II and

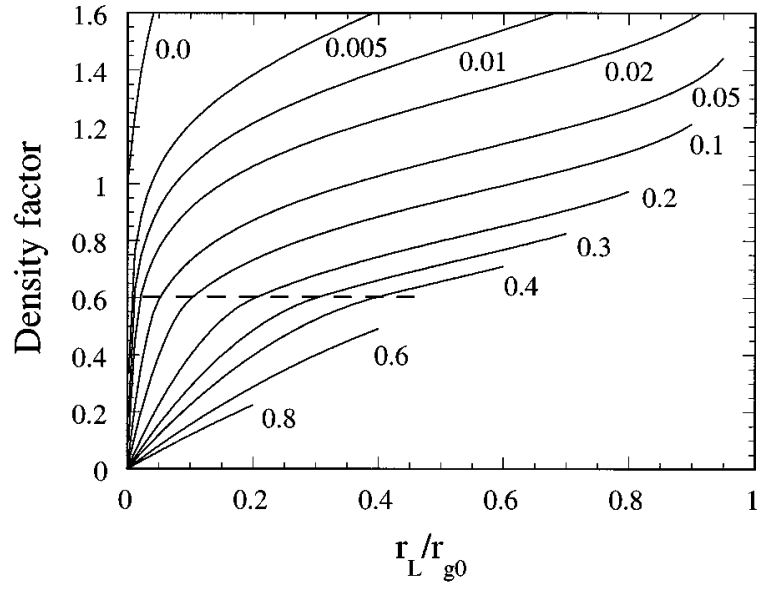

Fig. 10. Values of density reduction factor as a function of normalized Larmor radius $\left(r_{L} / r_{g 0}\right)$, for a number of different values of normalized guiding center spread $\left(a_{g} / r_{g 0}\right)$.

III. A better value for the average current density can be computed with (21)-(23) by writing the expected value as

$$
\bar{J}=\int_{0}^{\infty} J(r) \operatorname{pdf}(r) d r=\frac{2 \pi}{I} \int_{0}^{\infty}[J(r)]^{2} r d r
$$

and computing the average beam density $\bar{n}$ by dividing the result by $e v_{z}$. A convenient way to express the more accurate average density is as a multiplicative density correction factor $\left(\bar{n} / \bar{n}_{a n}\right)$ to the value expected from the uniform annulus model,

$$
\bar{n}_{a n}=\frac{I}{4 e \pi r_{g 0} r_{L} \mathrm{v}_{z}} .
$$

The values of this density correction factor for various values of $r_{L} / r_{g 0}$ and $a_{g} / r_{g 0}$, obtained by numerically integrating (27) using the current density profiles of (21)-(23), are plotted in Fig. 10. The density correction factor is significantly greater than 1 for small guiding center spreads and modest-to-large values of $r_{L} / r_{g 0}$, but the addition of significant spread quickly reduces the correction factor to below unity. Values below the horizontal dashed line are Case III geometries, and one can see that in this area, for small values of $r_{L} / r_{g 0}$, the curves in the plot become linear and pass through the origin. This corresponds to the uniform current density part of the beam (Region 2) dominating the overall current density profile, creating a nearly annular beam of radial width $2 a_{g}$, instead of $2 r_{L}$ as in the uniform annulus model. Under this limiting case the density correction factor is simply $r_{L} / a_{g}$. Electrostatic cyclotron instabilities in this limit have been studied by several authors [41], [43], [45], [46].

To give some specific examples, the density correction factor is about 0.75 for the beam of Fig. 2(a), and 0.52 for the beam of Fig. 2(b). Thus, the growth rate for electrostatic cyclotron modes in these two beams will be, respectively, 0.86 and 0.72 (taking the square root of the correction factor) of the growth rate expected from the uniform annulus model. These approximate reductions, since they appear in a spatial growth rate that is in an exponential, are significant in physically long systems. The density reduction factor is also an adiabatic invariant, so it remains valid when integrating the spatial growth rate through 
a slowly varying magnetic field to obtain a total growth of electrostatic cyclotron waves. Calculations performed using this methodology have shown that the peak growth in electrostatic cyclotron waves in a $35-\mathrm{GHz}$ gyroklystron employing the beam of Fig. 2(a) is reduced by over $3 \mathrm{~dB}$ at the $32-\mathrm{GHz}$ cyclotron frequency, as a result of the lower beam density [47]. During noise experiments on this device, electron cyclotron instabilities were not observed at the operating frequency. The lack of such instabilities was attributed to the existence of a small amount of velocity spread $\left(\sigma_{L} / r_{L 0}=4 \%\right)$, and the significant frequency detuning between the cyclotron frequency and the operating frequency, in addition to the reduced beam density [47]. However, a definitive experimental test of this hypothesis has not been performed, so a firm conclusion is not possible at present.

A final application for the improved current density profiles concerns the issue of mechanical clearance between the realistic beam and a cylindrical beam tunnel or rf interaction structure. In the idealized model without Larmor spread, the beam will have an outer edge at $r_{M}=r_{g 0}+a_{g}+r_{L}$, and an inner edge at $r_{m}=r_{g 0}-a_{g}-r_{L}$, so it would be a simple matter to specify the radial beam clearances and the axis alignment tolerance appropriately to avoid any interception. However, as can be seen in Fig. 8, any amount of Larmor spread creates extra tails beyond these "classical" beam edges, which complicates the problem. To make quantitative predictions of beam interception, one must include Larmor spread, because it is the sole contributor to the extra tails, rather than just a perturbation on a larger quantity.

Fortunately, it is possible to compute analytic expressions for the inner and outer tails, if one assumes $\sigma_{L} / r_{L 0}$ is small, below 0.15 or so, and if $\sigma_{L}<a_{g} / 4$. Considering first a simplified top-hat distribution in $r_{L}$, which defines $a_{L}=\sigma_{L} \sqrt{3}$ and $\operatorname{pdf}\left(r_{L}\right)=\left(2 a_{L}\right)^{-1}$ for $\left|r_{L}-r_{L 0}\right|<a_{L}$ and zero otherwise, and looking at the outer beam edge, one can write the current density as

$$
\begin{aligned}
J(r)=\frac{I}{4 \pi^{2} a_{g} r_{g 0}} & \left(\frac{1}{2 a_{L}}\right) \int_{r-r_{g 0}-a_{g}}^{r_{L 0}+a_{L}} d r_{L} \\
& \times\left(\pi-\theta_{1}-\sin ^{-1}\left(\frac{r_{L}}{r} \sin \theta_{1}\right)\right) .
\end{aligned}
$$

Here, the Region 3 profile has been used, and $\theta_{1}$ is given by (16) as before. When the Larmor spread is small, the value of $\theta_{1}$ is nearly $\pi$, so the expression for $\theta_{1}$ can be approximated as

$$
\theta_{1}=\cos ^{-1}(-1+K) \cong \pi-\sqrt{2 K}
$$

where $0<K \ll 1$ and is given by

$$
K=\left(r_{g 0}+a_{g}+r_{L}-r\right)\left(\frac{1}{r_{L 0}}+\frac{1}{r_{g 0}+a_{g}}\right) .
$$

Under these assumptions, the integration can be performed analytically to yield the current density in the outer tail region

$$
J(\delta)=\frac{I}{12 \pi^{2} a_{g} a_{L} r_{g 0}} \sqrt{\frac{2\left(r_{g 0}+a_{g}\right)}{r_{L 0}\left(r_{g 0}+a_{g}+r_{L 0}\right)}}\left(a_{L}-\delta\right)^{3 / 2}
$$

where $\delta=r-r_{M}$ is the radial distance outward from the "classical" (zero-Larmor spread) outer edge of the beam. The current density is plotted in Fig. 11 for the standard example values of $10 \mathrm{~A}$ beam current, $r_{g 0}=0.257 \mathrm{~cm}, r_{L 0}=0.0554$ $\mathrm{cm}, a_{g}=0.0212 \mathrm{~cm}$, and with a value of $a_{L}=0.0096 \mathrm{~cm}$ $\left(\sigma_{L} / r_{L 0}=10 \%\right)$. The gradual decay of current density is more realistic than the nearly vertical edge of (23), but it still reaches zero at a definite radius.

A more physically realistic description of the distribution of Larmor radius is a Gaussian about $r_{L 0}$, with standard deviation $\sigma_{L}$ [21], [31]-[33]. The expression for the outer tail region of the current density profile, assuming the Gaussian distribution, is given by

$$
\begin{aligned}
& J(x) \cong \frac{I}{4 \pi^{2} a_{g} r_{g 0}} \sqrt{\frac{\left(r_{g 0}+a_{g}\right) \sigma_{L}}{\pi\left(r_{g 0}+a_{g}+r_{L 0}\right) r_{L 0}}} \\
& \times \exp \left(-x^{2} / 2\right)\left(\frac{1}{2}\right)^{1 / 4} \Gamma(3 / 4) \\
& \times \exp \left\{-\left(x+\frac{\pi}{\Gamma(3 / 4)}\right.\right. \\
& \left.\left.\times\left[1-\sqrt{1+\left(\frac{\Gamma(3 / 4)}{\pi}(1 / 2)^{1 / 4} x\right)^{2}}\right]\right)\right\} .
\end{aligned}
$$

This equation was obtained similarly to the top-hat case, but the final integration could not be performed exactly, and was instead evaluated with the aid of a gamma function series expansion. The value of $\Gamma(3 / 4)$ is approximately 1.22541 , and $x=\delta / \sigma_{L}$. The current density given by (33) is also plotted in Fig. 11, for the same $10 \%$ value of $\sigma_{L} / r_{L 0}$. The falloff is initially similar to $\exp (-x)$ due to the second exponential factor, but at larger values of $x$ the decay becomes Gaussian. The corresponding formulas for the inner beam edge, useful for coaxial systems, are given by the outer edge expressions with $\hat{\delta}=r_{m}-r$ replacing $\delta$, and the ratio $\left(r_{g 0}-a_{g}\right) /\left(r_{g 0}-a_{g}-r_{L 0}\right)$ replacing $\left(r_{g 0}+\right.$ $\left.a_{g}\right) /\left(r_{g 0}+a_{g}+r_{L 0}\right)$ inside the leading square root term. This applies to both (32) and (33).

As a final topic, (33) can be used to compute the fractional amount of current intercepted by a cylinder surrounding the beam, $\left(I_{\text {int }} / I\right)$, where the radial clearance between $r_{M}$ and the cylinder wall $r_{w}$ is specified by $x_{w}=\left(r_{w}-r_{M}\right) / \sigma_{L}$. The resulting expression is

$$
\begin{aligned}
I_{\mathrm{int}} / I= & \frac{r_{L 0}}{a_{g} r_{g 0}} \\
& \times \sqrt{\left(r_{g 0}+a_{g}+r_{L 0}\right)\left(r_{g 0}+a_{g}\right)}\left(\frac{\sigma_{L}}{r_{L 0}}\right)^{3 / 2} \Phi\left(x_{w}\right)
\end{aligned}
$$

where $\Phi\left(x_{w}\right)$ is obtained by numerically integrating the exponential factors appearing in (33) over $x$ from $x_{w}$ to infinity, and multiplying the result by the numerical constants that appear in the equation. Values of $\Phi$ are listed in Table II

The beam interception equation is useful in choosing how many standard deviations of Larmor spread to allow between the beam and the wall. For example, in the Gaussian spread beam of Fig. 11, if $x_{w}=0,(34)$ indicates that $0.638 \%$ of the beam 


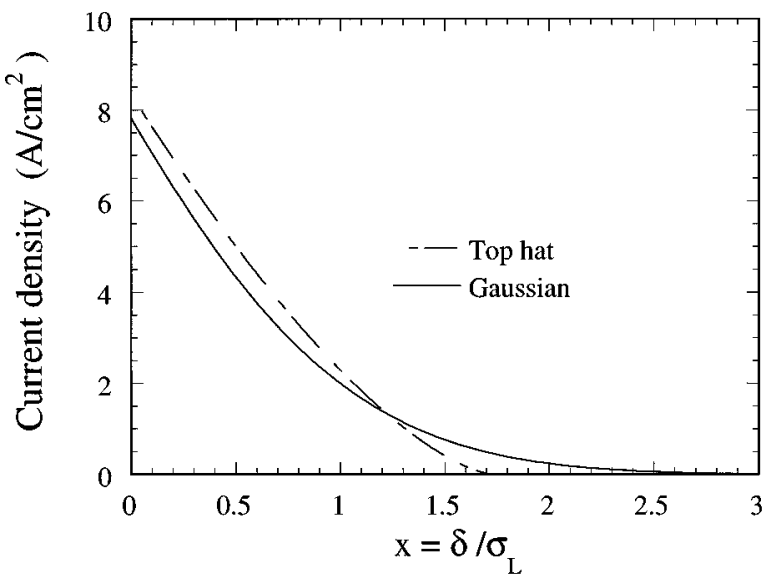

Fig. 11. Current density vs. radial position at the outer beam edge, including Larmor radius spread, for a top-hat, and a Gaussian distribution of Larmor radii. The calculations used $r_{g 0}=0.257 \mathrm{~cm}, a_{g}=0.0212 \mathrm{~cm}, r_{L 0}=0.0554 \mathrm{~cm}$, 10 A beam current, and $\sigma_{L} / r_{L 0}=10 \%$ for both distributions. The value of $\delta$ is the radial distance beyond the zero-Larmor-spread beam edge.

TABLE II

VALUE OF THE INTERCEPTION FUNCTION $\Phi(x)$

\begin{tabular}{c|ccccc}
\hline $\mathrm{x}$ & 0.0 & 0.2 & 0.4 & 0.6 & 0.8 \\
\hline 0 & 0.06496 & 0.04827 & 0.03498 & 0.02471 & 0.01700 \\
1 & 0.01139 & 0.007414 & 0.004692 & 0.002883 & 0.001719 \\
2 & $9.936 \times 10^{-4}$ & $5.565 \times 10^{-4}$ & $3.018 \times 10^{-4}$ & $1.584 \times 10^{-4}$ & $8.038 \times 10^{-5}$ \\
3 & $3.943 \times 10^{-5}$ & $1.869 \times 10^{-5}$ & $8.557 \times 10^{-6}$ & $3.782 \times 10^{-6}$ & $1.613 \times 10^{-6}$ \\
4 & $6.635 \times 10^{-7}$ & $2.632 \times 10^{-7}$ & $1.007 \times 10^{-7}$ & $3.713 \times 10^{-8}$ & $1.319 \times 10^{-8}$ \\
5 & $4.515 \times 10^{-9}$ & $1.489 \times 10^{-9}$ & $4.728 \times 10^{-10}$ & $1.446 \times 10^{-10}$ & $4.257 \times 10^{-11}$ \\
\hline
\end{tabular}

will be intercepted, or $63.8 \mathrm{~mA}$ for a 10-A beam. One can see from Table II that the falloff in interception current is very rapid with increasing $x_{w}$. In the same example, the interception will fall to $0.01 \mathrm{~mA}$ when $x_{w}$ is about 3.3 , which equates to a radial clearance of $0.18 \mathrm{~mm}$. In addition to specific calculations, (34) illustrates some interesting scalings that are not obvious without the formula; for instance, as the Larmor spread increases, the interception current with increase as the $3 / 2$ power of the Larmor spread, even if the wall is moved outward to maintain a constant value of $x_{w}$.

The usefulness of (34) and Table II in evaluating beam clearances depends on the relative magnitude of the Larmor spread compared to the achievable mechanical tolerances. If the tolerance in centering the wall around the beam plus the offset caused by angular misalignment over the length of the beam tunnel greatly exceeds the Larmor spread, then these mechanical issues totally dominate the situation and (34) is not very useful. This occurs in very high frequency, small guiding center, low Larmor spread guns such as the W-band gun of Fig. 2(b). On the other hand, for the $35-\mathrm{GHz}$ gyroklystron electron gun discussed throughout the paper, the formula is useful, although mechanical tolerances are often of comparable magnitude to the clearances computed with the formula. Finally, the interception equation becomes of great value in X-band devices, in which the required clearance associated with Larmor spread generally overwhelms clearances set by tolerance issues.

\section{SUMMARY}

Analytic expressions for the current density profiles in small orbit, spiraling electron beams, including guiding center spread, have been developed in this paper. The new expressions are much more realistic than the common approximations based on a uniform annulus model and a perfect turning-point model of the beam. The new expressions neglect space charge effects, and do not include a spread in Larmor radius, but these effects have little impact on the profiles of the beams produced by modern magnetron injection guns. The analytic current density profiles are useful in computing the thermal behavior of objects that intercept electron beams, such as supports for coaxial gyrodevices, or beam analyzer probes. The expressions are also useful in computing the average volume density of electrons within the beam, which is relevant for evaluating electrostatic cyclotron instability growth rates. The functional form of the analytic expressions could also be used in conjunction with beam analyzer scans to obtain least-squares fits, yielding experimental values of guiding center radius, guiding center spread, and mean Larmor radius. As a final topic, analytic expressions for the current density profile at the beam edges, including Larmor radius spread, were developed. The new analysis can be used to select a radial clearance between the beam and a beam tunnel or rf circuit, provided that the tolerances on mechanical alignment are considered simultaneously.

\section{ACKNOWLEDGMENT}

The author would like to thank K. T. Nguyen and J. J. Choi for providing EGUN simulation data for the W-band and $35 \mathrm{GHz}$ electron guns, respectively. The author also thanks B. G. Danly and B. Levush for overall discussions regarding this work, B. Hogan, W. Lawson, M. K. E. Flaherty, and J. Cheng for discussions on the topic of support rods in coaxial gyroklystrons, and T. M. Antonsen, Jr., and A. Fliflet for discussions on the topic of electrostatic cyclotron modes.

\section{REFERENCES}

[1] K. L. Felch et al., "Long pulse and CW tests of a $100 \mathrm{GHz}$ gyrotron with an internal, quasi optical converter," IEEE Trans. Plasma Sci., vol. 24, pp. 558-569, June 1996.

[2] K. L. Felch et al., "Characteristics and applications of fast wave gyrodevices," Proc. IEEE, vol. 87, pp. 752-781, May 1999.

[3] C. J. Edgcombe, Ed., Gyrotron Oscillators-Their Principles and Practice. London, U.K.: Taylor \& Francis, 1993.

[4] A. V. Gaponov-Grekhov and V. L. Granatstein, Eds., Applications of High Power Microwaves. Boston, MA: Artech House, 1994.

[5] V. L. Granatstein et al., "A quarter century of gyrotron research and development," IEEE Trans. Plasma Sci., vol. 25, pp. 1322-1335, Dec. 1997.

[6] M. Thumm, "State-of-the-art of high power gyro devices and free electron masers update 1999," Forschungszentrum , Karlsruhe, Germany, Scientific Rep. FZKA 6418, Feb. 2000.

[7] Y. Bykov et al., "The gyrotron system for ceramics sintering," in Microwaves: Theory Applications Materials Processing III, Ceramics Transactions. Westerville, OH: American Ceramic Soc., 1995, vol. 59, pp. 133-140.

[8] G. Link et al., "Sintering of advanced ceramics using a $30 \mathrm{GHz}, 10 \mathrm{~kW}$, CW industrial gyrotron," IEEE Trans. Plasma Sci., vol. 27, pp. 547-554, Apr. 1999.

[9] A. A. Tolkachev, "Gyroklystron-based $35 \mathrm{GHz}$ radar for observation of space objects," in Proc. 22nd Int. Conf. Infrared and Millimeter Waves, July 1997, pp. 37-38. 
[10] V. L. Granatstein and W. Lawson, "gyro amplifiers and candidate rf drivers for TeV linear colliders," IEEE Trans. Plasma Sci., vol. 24, pp. 648-665, June 1996.

[11] R. L. Schriever and C. C. Johnson, "A rotating beam waveguide oscillator," Proc. IEEE, vol. 54, pp. 2029-2030, Dec. 1966.

[12] A. V. Gaponov et al., "Induced synchrotron radiation of electrons in cavity resonators," JETP Lett., vol. 2, pp. 267-269, 1965.

[13] J. M. Baird and W. Lawson, "Magnetron injection gun (MIG) design for gyrotron applications," Int. J. Electron., vol. 61, pp. 953-967, Dec. 1986.

[14] W. B. Herrmannsfeldt, "Electron trajectory program," Stanford Linear Accelerator Center, Stanford Univ., , Stanford, CA, Rep. 226, Nov. 1979.

[15] _ - "Numerical design of electron guns and space charge limited transport systems," Nucl. Instrum. Methods. Phys. Res A, Accel. Spectrom. Detect. Assoc. Equip., vol. 187, pp. 245-253, Jan. 1981.

[16] V. K. Lygin, V. N. Manuilov, and S. E. Tsimring, "On methods of integral equations and auxiliary sources in the trajectory analysis of intense electron beams," Elektronika , vol. 401, pp. 36-38, July 1987.

[17] A. N. Kuftin et al., "Numerical simulation and experimental study of magnetron injection guns for powerful short wave gyrotrons," Int. J. Electron., vol. 72, pp. 1145-1151, May 1992.

[18] A. N. Kuftin et al., "Theory of helical electron beams in gyrotrons," Int J. Infrared Millim. Waves, vol. 14, pp. 783-816, Apr. 1993.

[19] T. M. Tran et al., "DAPHNE, a 2D axisymmetric electron gun simulation code," in Proc. 6th EPS-APS Int. Conf. Physics Computing, Lugano, Switzerland, August 1994, pp. 492-496.

[20] E. Borie, C. Gruber, and T. Westermann, "Calculation of MIG guns for gyrotrons using the BFCPIC code," Int. J. Electron., vol. 78, pp. 789-807, Apr. 1995.

[21] C. J. Edgcombe, "Sources of velocity spread in electron beams from magnetron injection guns," Int. J. Infrared Millim. Waves, vol. 16, pp. 83-97, Jan. 1995.

[22] A. Bondeson and T. M. Antonsen, "Space charge instabilities in gyrotron beams," Int. J. Electron., vol. 61, pp. 855-870, Dec. 1986.

[23] P. L. Meyer, Introductory Probability and Statistical Applications. Reading, MA: Addison-Wesley, 1970, ch. 4-6.

[24] S. Gasiorowicz, The Structure of Matter: A Survey of Modern Physics. Reading, MA: Addison-Wesley, 1979, p. 239.

[25] J. J. Choi et al., "Experimental investigation of a high power, two-cavity, 35 GHz gyroklystron amplifier," IEEE Trans. Plasma Sci., vol. 26, pp. 416-425, June 1998.

[26] J. P. Calame et al., "Experimental studies of bandwidth and power production in a three-cavity, $35 \mathrm{GHz}$ gyroklystron amplifier," Phys. Plasmas, vol. 6, pp. 285-297, Jan. 1999.

[27] M. Blank et al., "Demonstration of a $10 \mathrm{~kW}$ average power $94 \mathrm{GHz}$ gyroklystron amplifier," Phys. Plasmas, vol. 6, pp. 4405-4409, Dec. 1999.

[28] K. T. Nguyen et al., "Electron gun and collector design for $94 \mathrm{GHz}$ gyro amplifiers," IEEE Trans. Plasma Sci., vol. 26, pp. 799-813, June 1998

[29] K. R. Chu et al., "A 30 MW gyroklystron amplifier design for high energy linear accelerators," IEEE Trans. Plasma Sci., vol. 13, pp. 424-434, Dec. 1985.

[30] W. Lawson et al., "The design of a high peak power relativistic magnetron injection gun," Int. J. Electron., vol. 61, pp. 969-984, Dec. 1986.

[31] C. Liu, T. M. Antonsen Jr., and B. Levush, "Simulation of the velocity spread in magnetron injection guns," IEEE Trans. Plasma Sci, vol. 24, pp. 982-991, June 1996.

[32] S. E. Tsimring, "On the spread of velocities in helical electron beams," Radiophys. Quantum Electron., vol. 15, pp. 952-961, 1972.

[33] Y. Y. Lau, "Effects of cathode surface roughness on the quality of electron beams," J. Appl. Phys., vol. 61, pp. 36-44, Jan. 1987.
[34] J. M. Baird and A. C. Attard, "Gyrotron gun study report," Naval Research Laboratory, Washington, DC., Rep. TR-3-476, 1981.

[35] M. K. E. Flaherty et al., "Operation of a K-band second harmonic coaxial gyroklystron," J. Appl. Phys., vol. 76, pp. 4393-4398, Oct. 1994.

[36] W. Lawson et al., "High power operation of a three cavity X-band coaxial gyroklystron," Phys. Rev. Lett., vol. 81, pp. 3030-3033, Oct. 1998.

[37] J. M. Taccetti et al., "A Ka-band CHI-wiggler free electron maser: experimental results," Nuc. Instrum. Methods Phys. Res. A, Accel. Spectrom. Detect. Assoc. Equip., vol. 429, pp. 116-120, June 1999.

[38] M. E. Read et al., "Design of a $3 \mathrm{MW} 140 \mathrm{GHz}$ gyrotron with a coaxial cavity," IEEE Trans. Plasma Sci., vol. 24, pp. 586-595, June 1996.

[39] B. Piosczyk et al., "Coaxial cavity gyrotron with dual rf beam output," IEEE Trans. Plasma Sci., vol. 26, pp. 393-401, June 1998.

[40] L. Pages et al., "Energy loss, range, and bremsstrahlung yield for $10 \mathrm{keV}$ to $100 \mathrm{MeV}$ electron in various elements and chemical compounds," Atomic Data, vol. 4, pp. 1-127, 1972.

[41] H. Li and T. M. Antonsen Jr., "Space charge instabilities in gyrotron beams," Phys. Plasmas, vol. 1, pp. 714-729, Mar. 1994.

[42] S. Illy and E. Borie, "Investigation of beam instabilities in gyrotron oscillators using kinetic theory and particle-in-cell simulation," J. Plasma Phys., pt. 1, vol. 62, pp. 95-115, July 1999.

[43] K. R. Chen and K. R. Chu, "Study of noise amplification mechanism in gyrotrons," IEEE Trans. Microwave Theory Tech., vol. 34, pp. 72-79, Jan. 1986.

[44] T. M. Antonsen Jr. and W. M. Manheimer, "Shot noise in gyroklystrons," IEEE Trans. Plasma Sci, vol. 26, pp. 444-450, June 1998.

[45] J. L. Hirshfield, "Cyclotron harmonic maser," Int. J. Infared Millim. Waves, vol. 2, pp. 695-704, Apr. 1981.

[46] P. Charbit, A. Herscovici, and G. Mourier, "A partially self-consistent theory of the gyrotron," Int. J. Electron., vol. 51, pp. 303-330, Apr. 1981

[47] J. P. Calame et al., "Studies of electronic noise in gyroklystrons," Phys. Plasmas, vol. 7, pp. 2180-2185, May 2000.

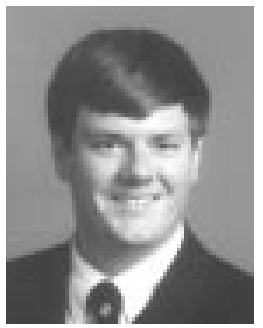

J. P. Calame (M'96) received the B.S., M.S., and $\mathrm{Ph} . \mathrm{D}$. degrees in electrical engineering from the University of Maryland, College Park, in 1985, 1986, and 1991 , respectively.

He performed part-time research on the electrical behavior of ionic crystals and ion-conducting polymers in the Physics Department, U.S. Naval Academy, Annapolis, MD, from 1980 to 1985 . His graduate research, from 1985 to 1991, involved the development of high peak power gyroklystrons. From 1991 to 1992 , was with the Naval Research Laboratory, Washington, DC, where he worked with microfabricated field emission electron sources and devices. From 1992 to 1997, he studied high-power microwave amplifiers, the microwave processing of materials, and the dielectric properties of ceramics at the Institute for Plasma Research, University of Maryland. Presently, he is with the Naval Research Laboratory, where he is developing high average power wideband millimeter wave amplifiers for radar applications. He is also studying the dielectric and thermal properties of composite ceramic materials and is investigating the intrinsic electronic noise in millimeter wave systems.

Dr. Calame received the 1991 APS Award for Outstanding Doctoral Dissertation Research in Beam Physics. 